\title{
Long-range and high-resolution correlation optical time-domain reflectometry utilizing an all optical chaotic source
}

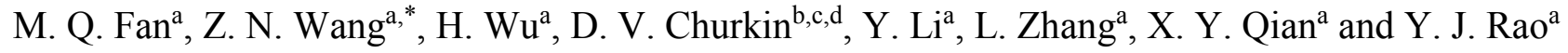 \\ ${ }^{a}$ Key Lab of Optical Fiber Sensing \& Communications, University of Electronic Science \\ \&Technology of China, Chengdu, Sichuan, China 611731 \\ ${ }^{b}$ Institute of Automation and Electrometry SB RAS, Novosibirsk 630090, Russia \\ 'Novosibirsk State University, Novosibirsk 630090, Russia \\ ${ }^{\mathrm{d}}$ Aston Institute of Photonic Technologies, Aston University, Birmingham, B4 7ET, UK \\ *znwang@uestc.edu.cn
}

\begin{abstract}
A high resolution optical time domain reflectometry (OTDR) based on an all-fiber chaotic source is demonstrated. We analyze the key factors limiting the operational range of such an OTDR, e.g., integral Rayleigh backscattering and the fiber loss, which degrade the optical signal to noise ratio at the receiver side, and then the guideline for counter-act such signal fading is discussed. The experimentally demonstrated correlation OTDR presents ability of $100 \mathrm{~km}$ sensing range and $8.2 \mathrm{~cm}$ spatial resolution (1.2 million resolved points), as a verification of the theoretical analysis. To the best of our knowledge, this is the first time that correlation OTDR measurement is performed over such a long distance with such high precision.
\end{abstract}

Keywords: Fiber measurements, Optical time domain reflectometry, Rayleigh scattering.

\section{INTRODUCTION}

Chaotic sources have attracted much attention due to its unique characteristics and the huge potential for various applications including physical random bit generation [1], secure communication [2], chaotic lidar [3] and so on. As an example of the applications of electrical driven chaotic source, in 2007, a cross-correlation OTDR [4] was proposed utilizing a pseudo-random pulse sequence as the signal. However, its measurement accuracy is still limited by the bottleneck of the pseudo-random modulation bandwidth, and it is difficult and costly to generate broadband electrical random codes. For the above reasons, electronic chaotic sources are hard to compete with optical chaotic sources which have advantages of wider bandwidth and faster chaotic dynamics.

Later on, Y. Wang et al. demonstrated a $6 \mathrm{~cm}$ spatial correlation OTDR based on a multi-GHz optical chaotic source utilizing LD laser [5]. This is the first proof-of-concept experiment of correlation OTDR by utilizing the optical chaotic source. However, that work only presented a $140 \mathrm{~m}$ detection ranging. There is always tradeoff between the spatial resolution and detection range.

In this work, we propose a long-range, high-precision correlation OTDR based on an all-fiber chaotic source. The setup for the source is same as our previous work [6]. The characteristics of the chaotic source were fully investigated in ref [6], and it shows potential to achieve millimeter-scale resolution OTDR. Here we further analyze the limiting factors for realizing long-range correlation OTDR, such as integral effect of weak Rayleigh backscattering and the fiber loss. The potential methods to overcome the bottleneck of operation range are proposed and analyzed. Specifically, the effects of distributed amplification on the enhancement of signal quality are analyzed. As a result, we experimentally demonstrate a correlation OTDR which has 100 kilometers fiber fault location range with $8.2 \mathrm{~cm}$ spatial resolution $(1.2$ million resolved points), with only $2 \mathrm{~ms}$ data acquisition time. To the best of our knowledge, this is the longest fiber fault location system based on the correlation OTDR technique.

\section{DISCUSSIONS FOR PERFORMANCE ENHANCEMENT OF CORRELATION OTDR}

As the major properties of the proposed source were studied, here we directly discuss for performance enhancement of correlation OTDR besides the method mentioned in ref [6], i.e., increasing sampling time. With the increase of sensing

Fifth Asia-Pacific Optical Sensors Conference, edited by Byoungho Lee, Sang-Bae Lee,

Yunjiang Rao, Proc. of SPIE Vol. 9655, 965548 - @ 2015 SPIE

CCC code: $0277-786 \mathrm{X} / 15 / \$ 18 \cdot$ doi: $10.1117 / 12.2203105$

Proc. of SPIE Vol. $9655965548-1$ 
range, OSNR of the detected signal would decrease thus it will be difficult to recognize the peaks among correlation trace. To extend the detection range of correlation OTDR, the signal quality must be improved.

Rayleigh scattering is the essential factor in this regime. At the receiver side, Rayleigh scattering components are an integral result. In another word, the fiber can be seen as a point-mirror with the integral Rayleigh scattering. When the fiber length is long enough, the Rayleigh scattering power will be significant [7], though the Rayleigh coefficient is very small ( $\varepsilon=4.3 \times 10^{-8} / \mathrm{m} @ 1550 \mathrm{~nm}$ ). We calculate the ratio of fiber-fault-reflected signal power to the Rayleighbackscattered noise, which could be expressed as : OSNR $=P_{i n} \cdot R \cdot \mathrm{e}^{-2 \alpha_{s} L} / P_{i n} \cdot \int_{0}^{L} \varepsilon \cdot e^{-2 \alpha_{s} l} d l$ where R stands for the reflectivity of the fiber fault, $\alpha_{s}$ represents the loss coefficient of signal (measured as $0.18 \mathrm{~dB} / \mathrm{km}$ ), $\mathrm{L}$ is the fiber length. The green solid curve in Fig. 1 shows the accumulated reflectivity taking both Rayleigh-backscattering and round-trip loss into account. In our model, we assume a 4\% Fresnel reflection to simulate the fiber fault. As shown in the insert of Fig. 1, the OSNR exponentially decreases with increased fiber length. From this calculation we see that if fiber length is $100 \mathrm{~km}$, the components of fiber-fault-reflected signal accounts very little $(-17.2 \mathrm{~dB})$ of the total power received by the detector.

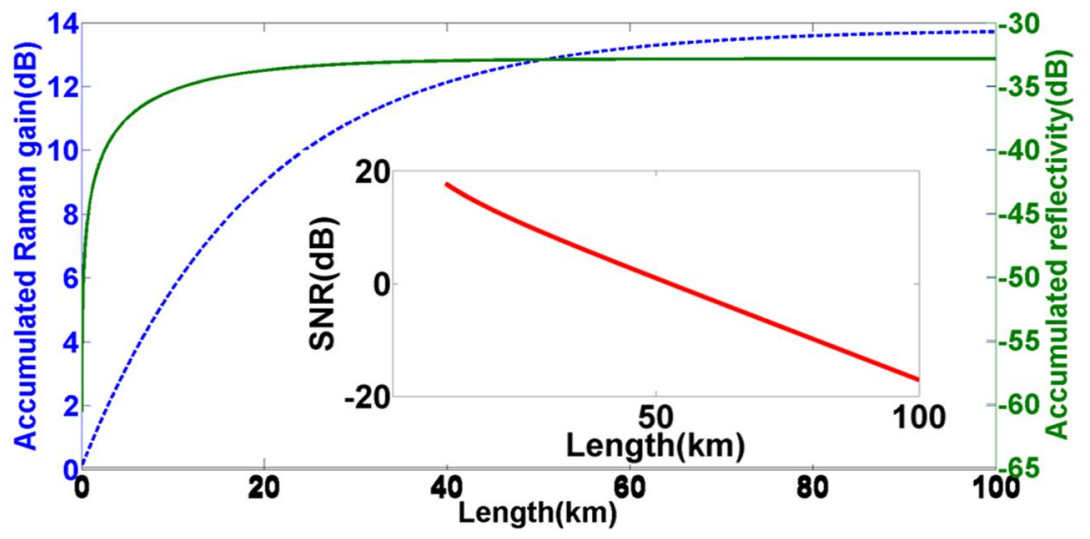

Fig. 1. Accumulated Raman gain over length and equivalent distribution reflectivity (insert: ratio of end-reflected signal power to Rayleigh-backscattered power along fiber without Raman amplification.

Here we show that the distributed amplification will perform much better than a pre-amplifier in front of the detector. A pre-amplifier such as an EDFA will boost the total optical power, but the OSNR would not enhance since the signal is actually buried in the in-band noise. On the other hand, when a distributed Raman amplifier is used, the intensity evolution of the chaotic probe light launched into the fiber can be calculated by the balanced steady equations [8]. By solving the equations, we calculate the ratio of fiber-fault-reflected signal power to the Rayleigh-backscattered power under Raman

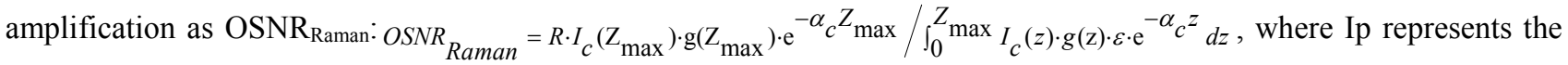
Raman pump intensity, Ic represents the chaotic probe light. $\mathrm{g}(\mathrm{z})$ stands for accumulated Raman gain distribution along fiber. We could obtain that OSNR will increase to $-6.6 \mathrm{~dB}$ under typical system parameters $(\mathrm{Ip}=26 \mathrm{dBm}$ and $\mathrm{Ic}=-5.5 \mathrm{dBm})$, which means a $10.6 \mathrm{~dB}$ enhancement compared with the case without Raman amplification $(-17.2 \mathrm{~dB})$. According to the above analysis, it can be concluded that Raman amplification is able to enhance the OSNR and therefore extend the sensing range of correlation OTDR significantly.

\section{EXPERIMENTAL DEMONSTRATION WITH CORRELATION OTDR}

Finally, we establish an experimental system following the schematic setup in ref [6]. The combination of 1550nm fiber Bragg grating (FBG) and a circulator (CIR1) is act as a narrow bandwidth filter. The $3 \mathrm{~dB}$ bandwidth of the filter is $0.26 \mathrm{~nm}$. After port3 of the CIR1, a 1:99 optical coupler is used to split the filtered light into two branches. One branch (1\%) is used as the reference light detected by a $1 \mathrm{GHz}$ photo-detector, while the other branch $(99 \%)$ transmission light acts as the chaotic probe light of OTDR. A Raman amplifier (RA) is used to enhance the signal quality. The fiber under test is $100 \mathrm{~km}$ standard single mode fiber (SMF) with $0.18 \mathrm{~dB} / \mathrm{km}$ loss at $1550 \mathrm{~nm}$. Because the reflected signal is very weak, we use a $1 \mathrm{GHz}$ avalanche photo-detector (APD) for signal detection. A $50 \mathrm{~cm} \mathrm{FC} / \mathrm{PC}$ jumper attached to the end of the $100 \mathrm{~km}$ SMF with FC/APC connector, both ends of which are used to emulate fiber-faults. 
We adjust all the parameters according to above analysis. The pump power of $\mathrm{SC}$ is $31.6 \mathrm{dBm}$, the power of $1 \%$ branch of the filtered chaotic light is $-25.5 \mathrm{dBm}$, while the $99 \%$ branch launched into RA is $-5.5 \mathrm{dBm}$. The RA power is set to $26 \mathrm{dBm}$. The total reflected light power detected by the APD is $-22.1 \mathrm{dBm}$. The signals of the two detectors are simultaneously recorded for $2 \mathrm{~ms}$ by a multi-channel oscilloscope with $5 \mathrm{GHz}$ sampling rate. The recorded data is processed with cross correlation algorithm and the fiber fault could be located by the correlation trace. Fig. 2(a) shows the normalized correlation OTDR trace. The position of the peak $(100.34080 \mathrm{~km})$ is corresponding to the open end of the FC/PC jumper. The SNR of our experiment result is $16.34 \mathrm{~dB}$ which is an excellent result considering the fiber length, suggesting that even longer range is achievable without any modification of the setup.
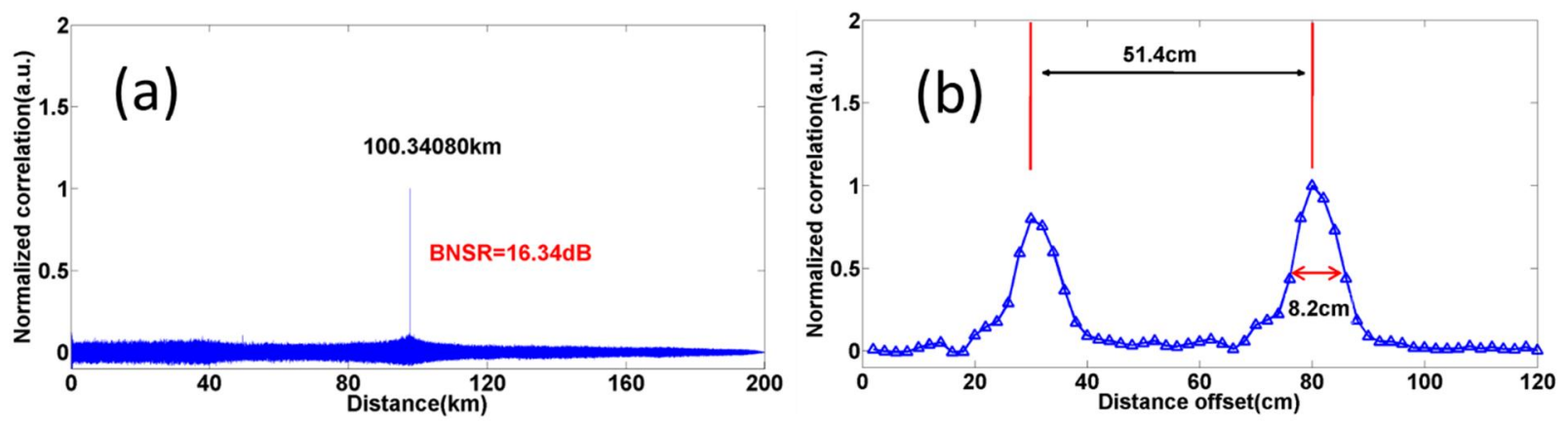

Fig. 2 (a) Experimental result of fault at 100.34080km; (b) magnified peaks of the correlation trace.

Fig. 2(b) shows the magnified correlation peaks. Because the FC/PC jumper has two flat- ends, the magnified correlation trace actually has two peaks. The two peaks are separated by $51.4 \mathrm{~cm}$ which represents the jumper length and the result is reasonably accurate. From the magnified trace, we also identify the spatial resolution of the system as $8.2 \mathrm{~cm}$.

\section{CONCLUSION}

In summary, a long-range and high-resolution correlation OTDR based on all-fiber chaotic source is proposed. The time domain properties and other correlation characteristics of such source studied in our previous work show the potential ability in high precision correlation OTDR. The key factors limiting the sensing range of correlation OTDR are analyzed, including integral effect of weak Rayleigh backscattering and the fiber loss. Based on the analysis, an instruction about how to achieve a long range correlation OTDR is presented. The key to extend sensing range is improving OSNR of the received signal, and we demonstrated that distributed amplification is a favorable approach. Finally, we realize cm-level spatial resolution and $100 \mathrm{~km}$ sensing range with $2 \mathrm{~ms}$ data acquisition time, which is a significant improvement compare to the conventional OTDR.

\section{ACKNOWLEDGMENTS}

This work is supported by Natural Science Foundation of China (61205048, 61290312), ERC Ultralaser Project (267763), Research Fund for the Doctoral Program of Higher Education of China (20120185120003), and PCSIRT (IRT1218), and the 111 project (B14039). The authors also thank Prof. Xingwen Yi in UESTC and Chengdu Best Xingbang Technology Limited for providing the oscilloscopes.

\section{REFERENCES}

[1] A. Uchida, K. Amano, M. Inoue, K. Hirano, S. Naito, H. Someya, I. Oowada, T. Kurashige, M. Shiki, S.Yoshimori, K. Yoshimura, and P. Davis, "Fast physical random bit generation with chaotic semiconductorlasers," Nat. Photonics 2, 728-732 (2008).

[2] A. Argyris, D. Syvridis, L. Larger, V. Annovazzi-Lodi, P.Colet, I. Fischer, J. Garcia-Ojalvo, C. R. Mirasso, L. Pesquera, and K. A. Shore, "Chaos-based communications at high bit rates using commercial fibre-optic links", Nature 438, 343-346 (2005)

[3] L. F. Y, L. J. M, “Chaotic lidar”, IEEE J. Sel. Top. Quantum Electron. 10, 991-997 (2004). 
[4] Y. Takushima and Y. C. Chung, "Optical reflectometry based on correlation detection and its application to the in-service monitoring of WDM passive optical network," Opt. Express 15, 5318-5326 (2007).

[5] Y. C. Wang, B. J. Wang, and A. B. Wang, "Chaotic correlation optical time domain reflectometry utilizing laser diode", IEEE Photon. Technol. Lett. 20, 1636-1638 (2008)

[6] M. Q. Fan, Z. N. Wang, H. Wua, D. V. Churkin, Y. Li, L. Zhang, X. Y. Qian and Y. J. Rao, "High resolution optical time-domain reflectometry based on correlation utilizing an all-fiber chaotic source", Asia Pacific Optical Sensors Conference 2015. International Society for Optics and Photonics, 2015.

[7] H. Wu, Z. N. Wang, M. Q. Fan, L. Zhang, W. L. Zhang, and Y. J. Rao, "Role of the mirror's reflectivity in forward-pumped random fiber laser," Opt. Express 23, 1421-1427 (2015).

[8] G. P. Agrawal, Nonlinear fiber optics, Academic Press, (2007). 